\title{
Psychometric evaluation of the Shona version of the Multidimensional Scale of Perceived Social Support Scale (MSPSS-Shona) in adult informal caregivers of patients with cancer in Harare, Zimbabwe
}

\author{
Jermaine M. Dambi, Lyster Tapera, Matthew Chiwaridzo, Cathrine Tadyanemhandu, \\ Clement Nhunzvi
}

College of Health Sciences, University of Zimbabwe, Harare, Zimbabwe

Correspondence: Mr Jermaine Dambi (jermainedambi@gmail.com)

\begin{abstract}
Background
Abstract

Providing care for a patient with cancer can negatively affect the health and psychosocial well-being of informal caregivers. However, social support has been enlisted as an essential buffer to stressful life events. There is now a greater call to routinely measure and provide support for caregivers and this is only feasible through use of validated outcome measures. The multidimensional scale of perceived social support (MSPSS) is one of the most commonly used social support outcome measure. Consequently, the MSPSS has been translated into several languages and validated across several populations. The aim of the present study was to translate the MPSS to Shona (Zimbabwean native language) and validate it in caregivers of patients with cancer.
\end{abstract}

Methods

The MSPSS was translated to Shona using a backward-forward translation method, pretested on a group of caregivers ( $\mathrm{n}=10$ ) before being administered to large sample $(\mathrm{N}=126)$ at Parirenyatwa Group of Hospitals. Both exploratory and confirmatory factor analysis were performed to assess the structural validity of the MSPSS-Shona version. Reliability was assessed using the Cronbach's alpha.

Results

Data for 120 caregivers were analysed. Most were females (69.2\%), had attained at least secondary education (81.7\%) and married (75\%). There was moderate evidence for structural validity for the 2 -factor model and excellent evidence for internal consistency as the scale yielded $\alpha=0.905$.

Conclusions

Despite moderate evidence for structural validity, the translation of MSPSS into native languages (e.g. MSPSS-Shona) in low resource settings can be deemed as "steps in the right direction" for evidence based practise in management of cancer. There is also need for further psychometric evaluation of the MSPSS-Shona.

\section{Introduction}

Cancer imposes a major burden worldwide, accounting for 8.2 million deaths (around 13\% of all deaths) globally as of the year 2012., ${ }^{1,2}$ The global burden is expected to exponentially increase to about 19.3 million new cases by the year 2025.,3 Unfortunately, more than $70 \%$ of all cancer-related deaths occur in resource constrained settings because of delayed diagnosis and poor treatment protocols due to a lack of resources. ${ }^{4-8}$ Further, the overall case fatality from cancer (ratio of mortality to incidence) is estimated to be $75 \%$ in low-income countries, compared with $46 \%$ in high income countries. ${ }^{7}$ The prevalence of cancer in Africa has also been on the upward course due to aging, population growth, poor health care facilities/systems and the general lifestyle changes which have consequently amplified the burden of noncommunicable diseases. ${ }^{2,9}$ The HIV/AIDS epidemic has further exacerbated the situation, ${ }^{2,4,9}$ for instance, statistics from Zimbabwe have revealed that $60 \%$ of patients with cancer are co-infected with HIV/AIDS. ${ }^{10}$

The increased burden of cancer has been unfortunately passed on to informal caregivers. ${ }^{11,12}$ For instance, due to the escalation of healthcare costs and the shift to communitybased treatment, there is now a greater reliance on informal caregivers in the management of long term health conditions such as cancer. ${ }^{11-13}$ "Informal carers are defined as carers who are not financially compensated for their services typically spouses, children, siblings or friends". ${ }^{13}$ As patients with cancer are often faced with a reduction/ deterioration in their physical functioning, psychosocial well-being and health-related quality of life (HRQoL), caregivers thus play an essential role in the management of cancer. ${ }^{5,12,14,15}$ For example, depending on the stage of and severity of the cancer, caregivers invariably play essential roles in the performance of day-to-day functional activities such as bathing, dressing, feeding among others. ${ }^{11-13}$ The role of caregivers is even pronounced in the palliative stage were the patient becomes more reliant on virtually every facet of life. ${ }^{11,13,15}$ Unfortunately, there is overwhelming, empirical evidence that the role of caregiving invariably results in a reduction in the caregivers' mental well-being and overall HRQoL., ${ }^{6,11,13,15,17,18}$ Evidence from several systematic reviews suggests that caregivers are more likely to report of depression, sleep problems, anxiety, decreased recreational time, fatigue, loneliness/decreased number of social contacts, weight loss, among other negative health outcomes. ${ }^{1,5,6,12,13,15,17-19}$ It is postulated that these negative effects are more likely to be greater in caregivers residing in low resource settings. ${ }^{5}$ 
To this end, there is now a greater call to improve the mental health of caregivers. ${ }^{1,5,6,12,13,17,18}$ As such, social support (SS) has been identified as an essential buffer to the strain associated with caregiving a patient with cancer. ${ }^{1,12,17,18}$ However, the development of context-specific interventions is very reliant upon use of patient-reported outcomes (PROs) which are both reliable and valid. ${ }^{13,15}$ This is only attainable if robust methodologies are applied in either development of context-specific PROs or during transcultural translations and adaptations of PROs..$^{15}$ Given how subjective and multidimensional SS is, various outcome measures have been developed with a few of these displaying robust psychometrical properties. ${ }^{13,15,17}$ To this end, the multidimensional scale of perceived social support scale (MSPSS) has evolved as one of the most commonly used SS outcome measure..$^{20-26}$ The MSPSS is a 12-item tool which quantifies the amount of SS one perceivably receives from family, friends and significant other/special person. ${ }^{27}$ Its brevity, cost (it is available free of charge) and demonstrated psychometric properties has led to its extensive usage and trans-cultural validation. ${ }^{28}$ The MSPSS has been previously validated in caregivers of patients with cancer and has been found to be both valid and reliable. ${ }^{1,29-38}$ It was therefore imperative to translate the MSPSS into Shona, a Zimbabwean native language. Secondly, it was also necessary to assess the acceptability, structural validity and reliability of the MSPSSShona version in adult caregivers of patients with cancer.

\section{Methods}

\section{Study design and translation}

The MSPSS was translated in accordance to the ISPORTranslation and Cultural Adaptation Group guidelines. ${ }^{39}$ Firstly, permission to translate the MSPSS to Shona was granted by the developers of the scale. Thereafter, the tool was translated from English to Shona by 2 independent, bilingual translators, i.e., both were fluent and proficient in English and Shona languages. The first translator had experience in translating PROs whereas the second translator had no prior experience with PROs translations. This was essential to get both a colloquial and conceptually equivalent translation. Thereafter, the 2 forward translations where then reconciled into 1 Shona translation by a third, independent translator. The reconciled forward translation was then translated backwards into English by another set of 2 independent translators who were not involved in the forward translation process. The 2 backward translations were then reconciled into 1 version by a fifth independent translator. Thereafter, the backward translation was compared with the original version of the MSPSS and no major differences were noted. The developer further confirmed the accuracy of the translation. Thereafter, the tool was pretested in a group of caregivers of adult patients with cancer ( $n=10)$ who were purposively selected from an outpatients' oncology clinic and these were not involved in the validation study. Participants were of different socio-economic and educational backgrounds. This was important for the evaluation of the appropriateness of the translation across different population sub-groups. The tool was self-administered and respondents were requested to comment on the cultural appropriateness, simplicity of the items and clarity of the scoring instructions. Thereafter, the tool was proofread by a clinician (physiotherapist) who had not been involved in the translation process to check for any typos before it could be administered to a larger sample.

\section{Setting}

Data were collected at Parirenyatwa Group of Hospitals (PGH), which is in Harare, Zimbabwe. PGH is the biggest referral centre in Zimbabwe and is a teaching hospital for the University of Zimbabwe. ${ }^{14}$ PGH provides specialist medical services and it has an oncology department where chemotherapy, radiotherapy, surgery and rehabilitation treatments are done. Patients receive services as either inpatients or outpatients. Oncology outpatient clinics are usually conducted on Mondays, Tuesdays and Thursdays.

\section{Participants}

Caregivers to outpatient patients with cancer presenting for oncology services were recruited as they were awaiting to receive services. Caregivers were included if they: were 18 years and above, unpaid in assuming the caregiving role, were involved in most of the caregiving (primary caregiver) of the patient diagnosed of cancer per doctors' notes and were willing to participate in the study. Were the patient was accompanied by multiple caregivers, only the primary caregiver was recruited. Caregivers who were not fluent in Shona, caregiving another relative with a long-term illness like cerebral palsy or had a confirmed diagnosis of a longterm conditions like diabetes, HIV/AIDS among others were similarly excluded as these would have confounded the study findings.

\section{Sampling and sample size calculation}

Literature posits that, ideally, 5 to 10 participants should be recruited per item for validation studies. ${ }^{40-41}$ Further, it is also recommended that at least 100 participants are needed for optimal results. ${ }^{40}$ We therefore, set to conveniently recruit at least 120 caregivers. Oversampling was done to cater for incomplete responses.

\section{Instrument}

The MSPSS is a 12-item questionnaire, which measures the amount of social support one receives from 3 sources: family (FAM), friends (FRE) and significant other/personal person (SO). Participants rate the amount of SS received on a 7-point Likert scale, which ranges from very strongly disagree (1) to very strongly agree (7). The scores are interpreted as, the higher the score, the greater the amount of perceived SS. ${ }^{27}$ The MSPSS was originally developed to measure social support in adolescents and has since been validated in both clinical and non-clinical samples.28 Due to its brevity and psychometric robustness, the MSPSS has been extensively translated and validated into several languages. ${ }^{25}$ The Shona version was developed and underwent initial validation testing for this study.

\section{Procedure and ethical considerations}

Institutional approval was granted by the clinical director at PGH. Thereafter, ethical clearance was sought and granted by the Joint Parirenyatwa and University of Zimbabwe Ethical Committee (Ref: JREC/269/16) and Medical Research Council of Zimbabwe (Ref: MRCZ/B/1172). Caregivers were approached at the oncology outpatient clinics which are done on Mondays, Tuesdays and Thursdays at PGH. The aims of the study were explained to the prospective participants. It was emphasised that participation was voluntary. Caregivers interested in participating were invited to a private room and were requested to sign consent forms in the presence of a witness. The researcher who had no clinical role in the oncology clinic then completed the purpose-design demographic questionnaire after screening 


\begin{tabular}{|c|c|c|c|}
\hline Variable & Attribute & Patients, n (\%) & Caregivers, $\mathbf{n}(\%)$ \\
\hline \multirow[t]{2}{*}{ Gender } & Male & $37(30.8)$ & $35(29.2)$ \\
\hline & Female & $83(69.2)$ & $85(70.8)$ \\
\hline ***Age & Mean (SD) & $59.6(15.1)$ & $41.64(13.3)$ \\
\hline \multirow[t]{9}{*}{ Type of cancer } & Cervical & $52(43.3)$ & \\
\hline & Prostate & $4(3.3)$ & \\
\hline & Breast & $18(15.0)$ & \\
\hline & Lung & $5(4.2)$ & \\
\hline & Colon & $10(8.3)$ & \\
\hline & Thyroid & $8(6.7)$ & \\
\hline & Rectal & $3(2.5)$ & \\
\hline & Gastric & $3(2.5)$ & \\
\hline & Others & $17(14.2)$ & \\
\hline \multirow[t]{4}{*}{ Type of treatment } & Chemotherapy & $69(57.5)$ & \\
\hline & Radiotherapy & $77(64.2)$ & \\
\hline & Surgery & $8(6.7)$ & \\
\hline & Brachytherapy & $5(4.2)$ & \\
\hline \multirow[t]{4}{*}{ Marital status } & Single & $7(5.8)$ & $21(17.5)$ \\
\hline & Divorced & $2(1.7)$ & 0 \\
\hline & Married & $73(60.8)$ & $90(75.0)$ \\
\hline & Widowed & $38(31.7)$ & $9(7.5)$ \\
\hline \multirow[t]{5}{*}{ Relationship to patient } & Parent & & $7(5.8)$ \\
\hline & Spouse & & $3(2.5)$ \\
\hline & Sibling & & $31(25.8)$ \\
\hline & Child & & $10(8.3)$ \\
\hline & Grandchild & & $52(43.3)$ \\
\hline \multirow[t]{3}{*}{ Highest level of education } & Primary & & $22(18.3)$ \\
\hline & Secondary & & $66(55.0)$ \\
\hline & Tertiary & & $32(26.7)$ \\
\hline \multirow[t]{4}{*}{ Employment status } & Formally employed & $14(11.7)$ & $31(25.8)$ \\
\hline & Self-employed & $3(2.5)$ & $12(10.0)$ \\
\hline & Student & $2(1.7)$ & $4(3.3)$ \\
\hline & Unemployed & $94(78.3)$ & $73(60.8)$ \\
\hline \multirow[t]{5}{*}{ Financial situation } & Very inadequate & & $60(50.0)$ \\
\hline & Inadequate & & $1(0.8)$ \\
\hline & Neutral & & $27(22.5)$ \\
\hline & Adequate & & $30(25.0)$ \\
\hline & Very adequate & & $2(1.7)$ \\
\hline
\end{tabular}

prospective participants for illegibility for inclusion. The caregivers then completed the MSPSS-Shona questionnaire and the questionnaires were collected on the same day.

\section{Data analysis}

Analysis of the study population descriptives and exploratory factor analysis (EFA) were performed using both SPSS (version 24) and STATISTICA (version 13.2). Confirmatory factor analysis (CFA) was performed using Stata (version 14). Cases with missing data were omitted listwise.

\section{Exploratory factor analysis}

Principal component analysis (PCA) was performed and this was essentially done in 3 stages. Firstly, data were checked for conformity with perquisite assumptions for EFA analysis. Normality was tested using the Shapiro Wilson Test (criterial value: $P>0.05)$, the identity of the correlation matrix was tested using the Bartlett Test of Sphericity (criterial value: $\mathrm{P}<0.05)$ and sampling adequacy was assessed using the Kaiser-Meyer-Oklin (KMO) measure (criterial value: KMO $>0.7) .{ }^{42}$ Thereafter, factors were extracted using the Kaiser criterion of 1 , meaning factors with eigenvalues $\geq 1$ were retained. The scree plot and Horn's parallel analysis methods were also used to determine the number of factors extracted for the unrotated factor solution. Lastly, the extracted factors underwent oblique rotation (Oblimin method) to improve the interpretability of the extracted solutions.

http://dx.doi.org/10.4314/mmj.v29i2.3
Table 2: Questionnaire item descriptions and mean response scores, $\mathbf{n}=120$

\begin{tabular}{lllcc}
\hline Factor & Item \# & Item description & Mean & Standard deviation \\
\hline Family & 8 & Family - problems & 3.76 & 0.91 \\
& 11 & Family - decisions & 3.67 & 1.11 \\
& 3 & Family - help & 3.65 & 1.35 \\
& 4 & Family - support & 3.55 & 1.29 \\
\hline Special person/ & 5 & Special - comfort & 3.78 & 1.08 \\
significant other & 1 & Special - need & 3.68 & 1.27 \\
& 2 & Special - joys & 3.80 & 1.11 \\
& 10 & Special - cares & 3.71 & 1.08 \\
\hline Friends & 6 & Friends - help & 3.37 & 1.28 \\
& 7 & Friends - wrong & 2.98 & 1.27 \\
& 9 & Friends - joys & 3.32 & 1.24 \\
& 12 & Friends - problems & 3.24 & 1.24 \\
\hline
\end{tabular}

\section{Confirmatory factor analysis}

The 12 items on the MSPSS constituted the observed endogenous variables and the 3 factors; family (FAM), friends (FRE) and special person/significant other (SO) constituted the latent endogenous variables. A nonrecursive structural equation model (SEM) was utilised for analysis. Three models, namely 1-, 2-, and 3-factor models were tested and it was hypothesised that the 3-factor model would provide a better fit. The following indices were used to assess the goodness of fit (gof) of the hypothesised models: Likelihood Ratio Chi-squared Test $\left(\mathrm{X}^{\mathrm{ms} 2}\right)$ - criterial value: $\mathrm{P}>0.05$, Root Mean Square Error of Approximation (RMSEA) -criterial value: $\leq 0.06$, Comparative Fit Index (CFI) - criterial value: $\geq 0.90$, Tucker-Lewis Index (TLI) - criterial value: $\geq 0.90$ and the Standardised Root Mean Square Residual (SRMR) criterial value $: \leq 0.06 .43$

\section{Reliability testing}

The reliability of the factors extracted and the total scale were calculated using the Cronbach's alpha $(\alpha)$. In accordance to Terwee et al., ${ }^{44}$ the minimal acceptable at both factor and scale level was set at 0.70 .

\section{Results}

\section{Demographic characteristics}

126 dyads of patients and caregivers were recruited. Six of the datasets had missing information on the MSPSS and these were omitted listwise. As illustrated in Table 1 below, most of the caregivers were females $(70.8 \%)$ and were mostly caregiving female patients $(69.2 \%)$. The mean patient's age was 59.6 (SD 15.1) years with most patients being diagnosed of cervical cancer and chemotherapy $(57.5 \%)$ and radiotherapy $(64.2 \%)$ were the most commonly received treatment modalities. Most of the caregivers were married $(75 \%)$, had attained at least secondary education $(81.7 \%)$, unemployed $(60.8 \%)$ and reported of severe financial challenges $(50 \%)$.

\section{Face validity}

Caregivers endorsed the cultural relevancy and clarity of the items on the MSPSS-Shona version. All the participants also attested to the clarity of the scoring instructions and layout of the questionnaire.

\section{EFA analysis results}

\section{Assumptions}

The data were normally distributed (Shapiro-Wilkson test $=$ $0.970, \mathrm{df}=120 \& \mathrm{P}=0.09)$, the Bartlett's test of sphericity was statistically significant $\left(\mathrm{X}^{2}(\mathrm{df} 66)=942.2, \mathrm{P}<0.001\right)$ with the KMO being 0.855 thus validating the suitability of the data for EFA. Further, there was no evidence of multicollinearity as the variables correlated reasonably, there were no coefficients greater than 0.9 on visual inspection of the correlation matrix. 


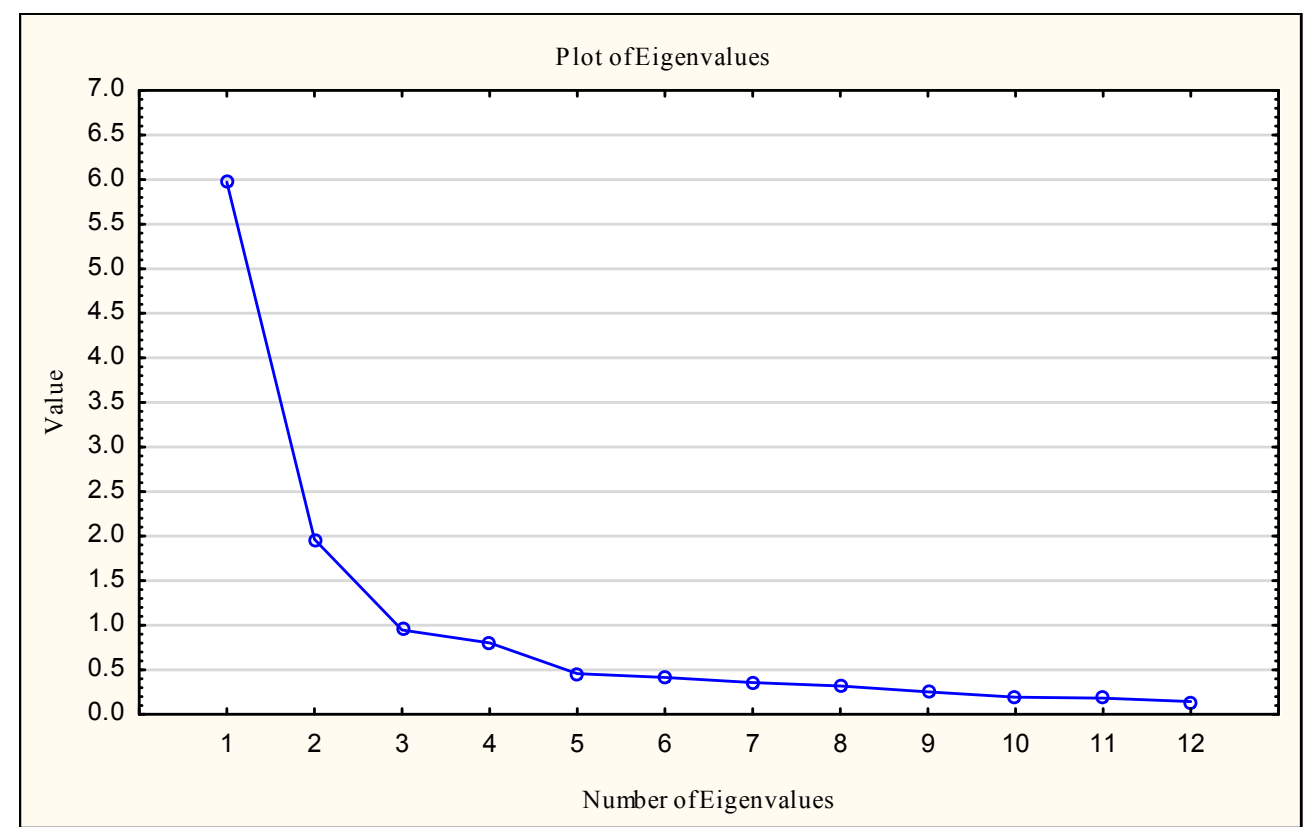

Figure 1: Scree plot for factor extraction

Table 3: Kaiser factor extraction results

\begin{tabular}{cccc|ccc}
\hline \multirow{2}{*}{ Component } & \multicolumn{3}{c}{ Extraction sums of squared loadings } \\
\cline { 2 - 7 } & Total & $\begin{array}{c}\text { \% of } \\
\text { Variance }\end{array}$ & $\begin{array}{c}\text { Cumulative } \\
\text { \% }\end{array}$ & Total & $\begin{array}{c}\text { \% of } \\
\text { Variance }\end{array}$ & $\begin{array}{c}\text { Cumulative } \\
\%\end{array}$ \\
\hline 1 & 5.972 & 49.764 & 49.764 & 5.972 & 49.764 & 49.764 \\
2 & 1.964 & 16.368 & 66.132 & 1.964 & 16.368 & 66.132 \\
3 & 0.948 & 7.896 & 74.029 & & & \\
\hline
\end{tabular}

Table 4: Pattern and structure matrices

\begin{tabular}{lcc|lcc}
\hline \multicolumn{2}{c|}{ Structure matrix } & \multicolumn{3}{c}{ Pattern matrix } \\
& Component & & \multicolumn{2}{c}{ Component } \\
& $\mathbf{1}$ & $\mathbf{2}$ & & $\mathbf{1}$ & $\mathbf{2}$ \\
\hline Family - help & 0.835 & & Family - help & 0.908 & \\
Family - decisions & 0.792 & & Family - support & 0.870 & \\
Special - need & 0.787 & & Family - decisions & 0.778 & \\
Family - support & 0.787 & & Special - need & 0.757 & \\
Special - comfort & 0.762 & 0.476 & Family - problems & 0.707 & \\
Special - joys & 0.755 & 0.436 & Special - joys & 0.692 & \\
Family - problems & 0.746 & & Special - comfort & 0.680 & \\
Special - cares & 0.627 & 0.539 & Special - cares & 0.488 & \\
Friends - joys & & 0.922 & Friends - joys & & 0.926 \\
Friends - problems & & 0.868 & Friends - problems & & 0.901 \\
Friends - wrong & & 0.861 & Friends - wrong & & 0.851 \\
Friends - help & 0.555 & 0.793 & Friends - help & & 0.679 \\
\hline
\end{tabular}

\section{Descriptives}

Friends were enlisted as the least source of social support and family were cited as the greatest source of support, the means (SD) for the specific items are outlined in Table 2.

\section{Factor retention}

Two factors accounted for $66.1 \%$ of the total variance according the Kaiser extraction method (Table 3) and this was also substantiated by inspection of the scree plot as there was an inflection between the third and fourth factors (Figure 1).

\section{Factor rotation and interpretation}

As illustrated in Table 4, 2 factors-friends and immediate family (family + significant other) - were retained upon inspection of both the structure and pattern matrices after Oblimin (oblique) rotation method was applied. Except for item 10 (There is a special person in my life who cares about my feelings), all items loaded fairly high as the factor loadings ranged from 0.679 to 0.926 . The friends' subscale had especially high factor loadings.

\section{Confirmatory factor analysis results}

\section{Goodness of fit comparison}

As illustrated in Table 5, the 1-factor model displayed the worst fit with the 3 -factor model presenting a much better goodness of fit (gof). Further, for both the 2- and 3-factor models, there was mixed evidence for gof as the results for the chi-square test and RMSEA were contradictory to those of the CFI, LFI and SRMR. As EFA supported a 2-factor model, the CFA results of the model are illustrated in Figure 3.

\section{Two-factor model output}

Figure 3 depicts the mean scores of the latent variables (items on the MSPSS) and how they relate to each other and to the original domains i.e. friends, significant other and family. The uniqueness of the specific variables is also presented. For example, for item 6 (my friends really try to help me) abbreviated FREhelp, the mean score for that item is 2.6 , it loads highly/highly correlated to the friend factor $(\mathrm{r}$ $=0.78$ ) and it uniquely contributes to $39 \%$ of the variance of the factor/domain. The greater the uniqueness, the less the relevant the item is to the overall model. 
Table 5: Comparison of goodness of fit for the 1-, 2-, and 3-factor models, $n=120$

\begin{tabular}{|c|c|c|c|}
\hline Index & 1-factor model & 2-factor model & 3-factor model \\
\hline Likelihood Ratio Chi-squared Test $\left(\mathbf{X}^{\mathrm{ms} 2}\right)$ & $\begin{array}{l}\mathrm{X}^{2}(\mathrm{df} 54)=402.2 \\
\mathrm{P}<0.001\end{array}$ & $\begin{array}{c}X^{2}(\text { df } 53)=217.9 \\
P<0.001\end{array}$ & $\begin{array}{l}X^{2}(\text { df } 51)=169.5 \\
\quad P<0.001\end{array}$ \\
\hline $\begin{array}{l}\text { Root mean squared error of } \\
\text { approximation (RMSEA) }(90 \% \mathrm{CI})\end{array}$ & $0.233(0.21$ to 0.254$)$ & $0.162(0.140$ to 0.184$)$ & $0.14(0.117$ to 0.163$)$ \\
\hline Comparative fit index (CFI) & 0.623 & 0.822 & 0.872 \\
\hline Tucker-Lewis index (LFI) & 0.540 & 0.778 & 0.834 \\
\hline $\begin{array}{l}\text { Standardised root mean squared residual } \\
\text { (SRMR) }\end{array}$ & 0.135 & 0.091 & 0.088 \\
\hline
\end{tabular}

Table 6: Reliability coefficients for MSPSS-Shona components

\begin{tabular}{l|ccccc}
\hline & \multicolumn{5}{|c}{ Scale } \\
\cline { 2 - 6 } & SO & FRE & FAM & FAM \& SO & Scale level \\
\hline $\begin{array}{l}\text { Cronbach's } \\
\text { alpha }(\boldsymbol{\alpha})\end{array}$ & 0.847 & 0.897 & 0.857 & 0.897 & 0.905 \\
\hline
\end{tabular}

$\mathrm{SO}=$ special person/significant other; FRE $=$ friends; FAM = family

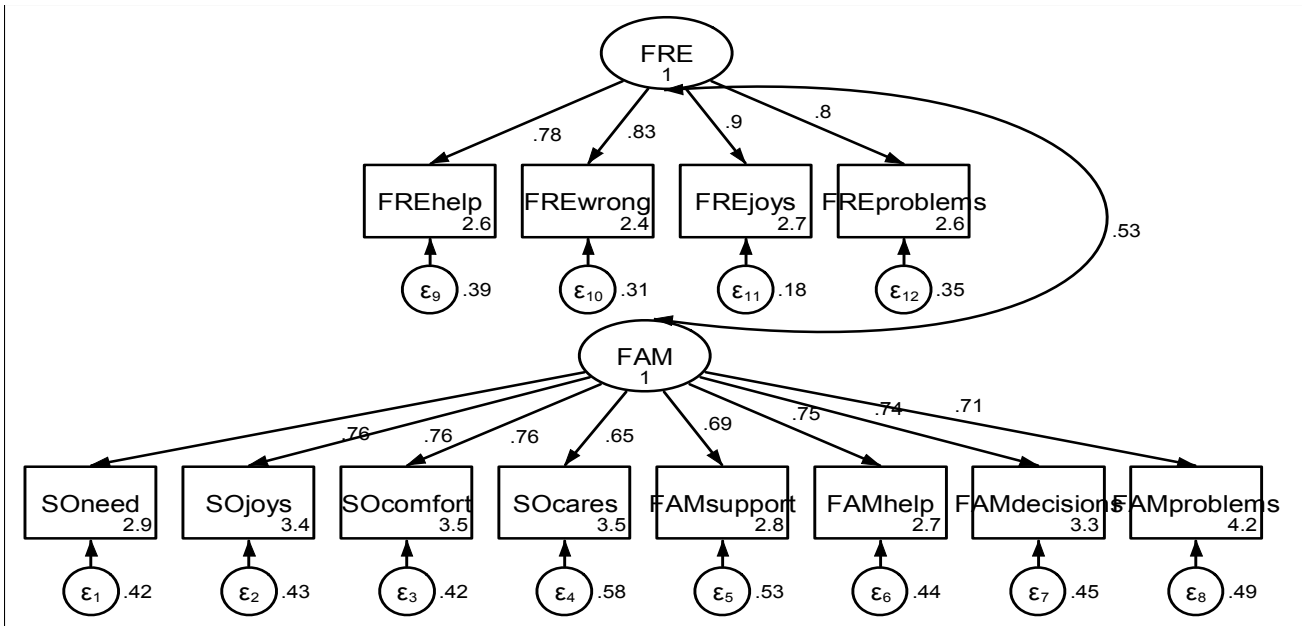

Figure 2: Two-factor structural equation model pathway diagram

MSPSS-Shona scales: $\mathrm{SO}$ = special person/significant other; FAM = family 2-factor structures. It is postulated that differences in culture influence ones' perception of the amount of social support rendered. ${ }^{20,45,46}$ In collectivistic cultures in Asian countries, such as China and Pakistan, it is difficult for one to precisely discriminate between the hypothesised 3 sources of social support. ${ }^{45,46}$ Questions for the significant other (SO) factor are phrased as "... there is someone special...". Due to cultural and linguistic differences, it could have been difficult for native Shona speakers to distinguish between family and "special person" as the 2 are used interchangeably in the Shona culture. This is also in keeping with outcomes of the validation of the Hausa version of the MSPSS which also has an almost similar culture. ${ }^{48}$ Further, the Shona language has 5 other dialects ${ }^{49}$ and this could have led to the convergence of the participants' responses on the family and significant subscales. This is only but speculation which may need further empirical investigation.

Given the high literacy level of the study participants, it was most unlikely that the respondents did not accurately respond to the questionnaire. Furthermore, scale and factor-level

\section{Reliability}

As illustrated in Table 5, the friends sub-scale yielded the greatest reliability $(\alpha=0.897$ ) and the scale level reliability was Cronbach's alpha 0.905 .

\section{Discussion}

To the best of our knowledge, this is the first study to validate the MSPSS in adult caregivers of patients with cancer in the African setting. Based on the robust translation procedure employed and feedback from the caregivers, there is evidence suggestive of the accuracy in the translation process. However, we did not replicate the original 3-factor structure as the MSPSS-Shona version yielded a 2-factor structure after undergoing both EFA and CFA. Further, there was mixed evidence for model goodness of fit indices. We postulate that the discrepancy could be accounted for by differences in culture and or methodological quality of the validation study.

Elsewhere, the traditional Chinese ${ }^{45}$ and Urdu (Pakistani) ${ }^{46}$ versions of the MSPSS yielded a 1-factor structure, and both the Spanish ${ }^{47}$ and Hausa (Nigerian) ${ }^{48}$ translations yielded reliability findings also support the lack of differentiation between family and special person as sources of support. For instance, the internal consistency for immediate family (SO and FAM combined) was $\alpha=0.897$ which was greater than that of SO $(\alpha=0.847)$ and FAM $(\alpha=0.856)$ sub-scales computed separately. Given that most of the participants were married $(75 \%)$, it can be argued that spouses would be regarded as family and vice versa. This is also consistent with findings from the validation of the MSPSS in Arabian women. ${ }^{50}$

The MSPSS has been previously validated in 3 African countries i.e. in Malawi, ${ }^{51}$ Nigeria, $^{48}$ and Uganda. ${ }^{52}$ The 3-factor structure was replicated for the Malawi (Chichewa and Chiyao versions) and Uganda (Luganda version) translations. However, the aforementioned studies had some methodological limitations as measured by the COnsensus-based Standards for the selection of health status Measurement Instruments (COSMIN) checklist, ${ }^{53}$ which potentially limits their external validity. For example, only 1 backward translation was performed for the Hausa, Malawi Med J. 2017 Jun;29(2):89-96 
Chichewa, and Chiyao versions. ${ }^{48,51}$ Additionally, only EFA was performed an incorrect factor rotation method (orthogonal rotation) was utilised for the validation of the Luganda version..$^{52}$ Given these limitations, the validity and reliability thus becomes questionable. Moreover, both EFA and CFA are a perquisite to confirm the structural validity of a translated and adapted PRO. ${ }^{53,54}$ More so, none of the studies involved assessment of SS in caregivers and this poses challenges in comparability with the present study.

\section{Conclusions}

Participants were conveniently selected which is a limitation as this violates the assumptions for structural validity testing. Although there was moderate evidence for structural validity, given the robust translation process and the high reliability indices yielded, the findings are suggestive that the MSPSS-Shona could be a valid and reliable social support outcome measure in caregivers of adult patients with cancer in the Zimbabwean setting. However, there is further need to administer the MSPSS-Shona in a larger sample and compare the outcomes with randomly selected healthy, adult Zimbabweans to minimise confounding thus ensuring methodological rigor. Future qualitative studies are also warranted to better understand the perceived meaning and importance of social support in native Shona speakers. Further studies evaluating other psychometric properties such as test-retest reliability, construct validity, and responsiveness are also needed. Despite these, moderate evidence for structural validity, the translation of MSPSS into native languages (e.g., MSPSS-Shona) in low-resource settings can be deemed as an essential "step in the right direction" for evidence-based practise in management of cancer.

\section{Acknowledgements}

Special thanks go to the caregivers and patients with cancer for their voluntary participation.

\section{Funding}

The MSPSS translation is part of the primary authors' $\mathrm{PhD}$ work at the University of Cape Town. The work is being funded by The African Mental Health Research Initiative (AMARI). AMARI is a consortium of 4 African universities whose overall goal is to build excellence in leadership, training and science amongst African scholars in mental, neurological and substance use (MNS) research in Ethiopia, Malawi, South Africa and Zimbabwe. AMARI, at the University of Zimbabwe College of Health Sciences (UZCHS) secured funding from the Wellcome Trust through the Developing Excellence in Leadership and Science (DELTAS) Africa initiative. The DELTAS Africa Initiative is an independent funding scheme of the African Academy of Sciences (AAS)'s Alliance for Accelerating Excellence in Science in Africa (AESA) and supported by the New Partnership for Africa's Development Planning and Coordinating Agency (NEPAD Agency) with funding from the Wellcome Trust [DEL-15$01]$ and the UK government. The views expressed in this publication are those of the author(s) and not necessarily those of AAS, NEPAD Agency, Wellcome Trust or the UK government.

\section{Competing interests}

All authors declare that they have no competing interests related to this work.

\section{References}

1. Ng CG, Mohamed S, See MH, et al. Anxiety, depression, perceived social support and quality of life in Malaysian breast cancer patients: a 1-year prospective study. Health Qual Life Outcomes. 2015;13(1):1-9. doi:10.1186/s12955-015-0401-7.

2. Thun MJ, DeLancey JO, Center MM, Jemal A, Ward EM. The global burden of cancer: Priorities for prevention. Carcinogenesis. 2009;31(1):100-110. doi:10.1093/carcin/bgp263.

3. The Centers for Disease Control and Prevention. Cancer. At a glance fact sheets. https://www.cdc.gov/chronicdisease/resources/ publications/aag/dcpc.htm. Published 2016. Accessed March 14, 2017.

4. Morhason-Bello IO, Odedina F, Rebbeck TR, et al. Challenges and opportunities in cancer control in Africa: A perspective from the African Organisation for Research and Training in Cancer. Lancet Oncol. 2013;14(4):e142-e151. doi:10.1016/S1470-2045(12)70482-5.

5. Li Q, Xu Y, Zhou H, Loke AY. Factors influencing the health-related quality of life of Chinese advanced cancer patients and their spousal caregivers: a cross-sectional study. BMC Palliat Care. 2016;15:72. doi:10.1186/s12904-016-0142-3.

6. Krug K, Miksch A, Peters-Klimm F, Engeser P, Szecsenyi J. Correlation between patient quality of life in palliative care and burden of their family caregivers: a prospective observational cohort study. BMC Palliat Care. 2016;15(1):4. doi:10.1186/s12904-016-0082-y.

7. Kingham TP, Alatise OI, Vanderpuye V, et al. Cancer Control in Africa 3 Treatment of cancer in sub-Saharan Africa. Lancet Oncol. 2013;14(4):e158-e167. doi:10.1016/S1470-2045(12)70472-2.

8. World Health Organization. The Global Burden of Cancer. http:// www.who.int/mediacentre/factsheets/fs297/en/. Published 2017. Accessed June 19, 2017.

9. Jemal A, Bray F, Forman D, et al. Cancer burden in Africa and opportunities for prevention. Cancer. 2012;118(18):4372-4384. doi:10.1002/cner.27410.

10. Nyakabau AM. Priorities for Cancer Prevention and Control in Zimbabwe. Cancer Control. http://www.cancercontrol.info/cc2014/ priorities-for-cancer-prevention-and-control-in-zimbabwe/. Published 2014. Accessed February 16, 2017.

11. Ugur O, Elcigil A, Arslan D, Sonmez A. Responsibilities and difficulties of caregivers of cancer patients in home care. Asian Pacific J Cancer Prev. 2014;15(2):725-729. doi:10.7314/APJCP.2014.15.2.725.

12. Hudson PL, Remedios C, Thomas K. A systematic review of psychosocial interventions for family carers of palliative care patients. BMC Palliat Care. 2010;9:17. doi:10.1186/1472-684X-9-17.

13. Michels CTJ, Boulton M, Adams A, Wee B, Peters M. Psychometric properties of carer-reported outcome measures in palliative care: A systematic review. Palliat Med. 2016;30(1):23-44. doi:10.1177/0269216315601930.

14. Dambi JM , Makotore FG M, Kaseke F. The Impact of Caregiving a Child with Cancer: A Cross Sectional Study of Experiences of Zimbabwean Caregivers. J Palliat Care Med. 2015;5(5). doi:10.4172/2165-7386.1000230.

15. Shilling V, Matthews L, Jenkins V, Fallowfield L. Patient-reported outcome measures for cancer caregivers: a systematic review. Qual Life Res. 2016;25(8):1859-1876. doi:10.1007/s11136-016-1239-0.

16. Awadalla AW, Ohaeri JU, Gholoum A, Khalid AO a, Hamad HM a, Jacob A. Factors associated with quality of life of outpatients with breast cancer and gynecologic cancers and their family caregivers: a controlled study. BMC Cancer. 2007;7:102. doi:10.1186/1471-2407-7102.

17. Dam AEH, De Vugt ME, Klinkenberg IPM, Verhey FRJ, Van Boxtel MPJ. A systematic review of social support interventions for caregivers of people with dementia: Are they doing what they promise? Maturitas. 2016;85(2016):117-130. doi:10.1016/j.maturitas.2015.12.008. 
18. Tang WPY, Chan CWH, So WKW, Leung DYP. Web-based interventions for caregivers of cancer patients : A review of literatures. Asia-Pacifi c J Oncol Nurs. 2014;1(1). doi:10.4103/2347-5625.135811.

19. Mbah O, Ford JG, Qiu M, et al. Mobilizing social support networks to improve cancer screening: the $\mathrm{COACH}$ randomized controlled trial study design. BMC Cancer. 2015;15:907. doi:10.1186/s12885-0151920-7.

20. Ekbäck M, Benzein E, Lindberg M, Årestedt K. The Swedish version of the multidimensional scale of perceived social support (MSPSS) - a psychometric evaluation study in women with hirsutism and nursing students. Health Qual Life Outcomes. 2013;11(1):168. doi:10.1186/1477-7525-11-168.

21. Pedersen SS, Spinder H, Erdman R a M, Denollet J. Poor perceived social support in implantable cardioverter defibrillator (ICD) patients and their partners: cross-validation of the multidimensional scale of perceived social support. Psychosomatics. 2009;50(5):461-467. doi:10.1016/S0033-3182(09)70838-2.

22. Bagherian-Sararoudi R, Hajian A, Bahrami Ehsan H, Sarafraz MR, Zimet GD. Psychometric properties of the Persian version of the multidimensional scale of perceived social support in Iran. Int J Prev Med. 2013;4(11):1177-1181.

23. Ng CG, Amer Siddiq AN, Aida SA, Zainal NZ, Koh OH. Validation of the Malay version of the Multidimensional Scale of Perceived Social Support (MSPSS-M) among a group of medical students in Faculty of Medicine, University Malaya. Asian J Psychiatr. 2010;3(1):3-6. doi:10.1016/j.ajp.2009.12.001

24. Guan NC, Seng LH, Hway Ann AY, Hui KO. Factorial validity and reliability of the Malaysian simplified Chinese version of Multidimensional Scale of Perceived Social Support (MSPSS-SCV) among a group of university students. Asia Pac J Public Health. 2015;27(2):225-231. doi:10.1177/1010539513477684.

25. Hannan J, Alce M, Astros A. Psychometric properties of the newly translated creole multidimensional scale of perceived social support (MSPSS) and perceived adequacy of resource scale (PARS) and the relationship between perceived social support and resources in Haitian mothers in th. BMC Psychol. 2016;4:7. doi:10.1186/s40359-016-01138.

26. Hamza A, Mohsein NAA, Yim L siew. Measuring percieved social support in stroke survivors : linguistic validation of the Multidimensional Scale of Percieved Social Support ( MSPSS ) in Hausa ( Nigerian ) language. South Africa J Occup Ther. 2012;42(830).

27.Zimet GD, Dahlem NW, Gimet SG, Farley GK. The Multidimensional Scale of Percieved Social Support. J Pers Assess. 1988;52(1):30-41.

28. Hardan-Khalil K, Mayo AM. Psychometric Properties of the Multidimensional Scale of Perceived Social Support. Clin Nurse Spec. 2015;(September/October):258-261. doi:10.1023/A:1005109522457.

29. Kuscu MK, Dural U, Önen P, et al. The association between individual attachment patterns, the perceived social support, and the psychological well-being of Turkish informal caregivers. Psychooncology. 2009;18(9):927-935. doi:10.1002/pon.1441.

30. Yakar HK, Pinar R. Reliability and Validity of Turkish Version of the Caregiver Quality of Life Index Cancer Scale. Asian Pacific J Cancer Prev. 2013;14(7):4415-4419. doi:10.7314/APJCP.2013.14.7.4415.

31. Okcin F, Karadakovan A. Reliability and validity of the quality of life -Family version (QOL-FV) in Turkish family caregivers of patients with cancer. Asian Pacific J Cancer Prev. 2012;13(9):4235-4240. doi:10.7314/APJCP.2012.13.9.4235.

32. Nik Jaafar NR, Selamat Din SH, Mohamed Saini S, et al Clinical depression while caring for loved ones with breast cancer. Compr Psychiatry. 2014;55(SUPPL. 1):S52-S59. doi:10.1016/j. comppsych.2013.03.003.

33. Han Y, Hu D, Liu Y, et al. Coping styles and social support among depressed Chinese family caregivers of patients with esophageal cancer. Eur J Oncol Nurs. 2014;18(6):571-577. doi:10.1016/j.ejon.2014.07.002.
34. Han Y, Yuan J, Luo Z, et al. Determinants of hopelessness and depression among Chinese hospitalized esophageal cancer patients and their family caregivers. Psychooncology. 2013;22(11):2529-2536. doi:10.1002/pon.3315.

35. Yoon HS, Lim YO, Choi K, Kim Y, Lee HJ. Depression of older adults with cancer in Korea. Clin Gerontol. 2015;38(5):395-411. doi:10 .1080/07317115.2015.1067273.

36. Rokach A, Findler L, Chin J, Lev S, Kollender Y. Cancer patients, their caregivers and coping with loneliness. Psychol Health Med. 2013;18(2):135-144. doi:10.1080/13548506.2012.689839.

37. Morse KD, Gralla RJ, Petersen J a, Rosen LM. Preferences for cancer support group topics and group satisfaction among patients and caregivers. J Psychosoc Oncol. 2014;32(1):112-123. doi:10.1080/0734 7332.2013.856058.

38. Gilbar O. Parent caregiver adjustment to cancer of an adult child. J Psychosom Res. 2002;52(5):295-302. doi:10.1016/S00223999(01)00259-8.

39. Wild D, Grove A, Martin M, et al. Principles of Good Practice for the Translation and Cultural Adaptation Process for Patient-Reported Outcomes (PRO) Measures: report of the ISPOR Task Force for Translation and Cultural Adaptation. Value Health. 2005;8(2):94-104. doi:10.1111/j.1524-4733.2005.04054.x.

40. de Winter JCF, Dodou D, Wieringa PA. Exploratory Factor Analysis With Small Sample Sizes With Small Sample Sizes. Multivariate Behav Res. 2009;44(April):147-181. doi:10.1080/00273170902794206.

41. Pearson RH, Mundform DJ. Recommended Sample Size for Conducting Exploratory Factor Analysis on Dichotomous Data. J Mod Appl Stat Methods. 2010;9(2):359-368.

42. Costello AB, Osbourne JW. Best practices in exploratory factor analysis: Four recommendations for getting the most from your analysis. Pract Assessment, Res Eval. 2005;10(7):1-9. doi:10.1.1.110.9154.

43. Schreiber J, Nora A. Reporting structural equation modeling and confirmatory factor analysis results: A review. J Educ Res. 2006;6(99):323-338. doi:10.3200/JOER.99.6.323-338.

44. Terwee CB, Bot SDM, de Boer MR, et al. Quality criteria were proposed for measurement properties of health status questionnaires. J Clin Epidemiol. 2007;60(1):34-42. doi:10.1016/j.jclinepi.2006.03.012.

45. Chou K-L. Assessing Chinese adolescents' social support: the multidimensional scale of perceived social support. Pers Individ Dif. 2000;28(2):299-307. doi:10.1016/S0191-8869(99)00098-7.

46. Akhtar A, Rahman A, Husain M, Chaudhry IB, Duddu V, Husain N. Multidimensional scale of perceived social support: psychometric properties in a South Asian population. J Obstet Gynaecol Res. 2010;36(4):845-851. doi:10.1111/j.1447-0756.2010.01204.x.

47. Cobb C, Xie D. Structure of the multidimensional scale of perceived social support for undocumented Hispanic immigrants . Hisp J Behav Sci. 2015;37(2):274-281. doi:10.1177/0739986315577894.

48. Mohammad AH, Al Sadat N, Yim LS, Chinna K. Validity and Reliability of the Hausa Version of Multidimensional Scale of Perceived Social Support Index. Iran Red Crescent Med J. 2015;17(2):0-6. doi:10.5812/ircmj.18776.

49. Mhute I. Standardisation a Considerable Force behind Language Death : A Case of Shona. J Educ Pract. 2016;7(9):62-65.

50. Aroian K, Templin TN, Ramaswamy V. Adaptation and psychometric evaluation of the Multidimensional Scale of Perceived Social Support for Arab immigrant women. Health Care Women Int. 2010;31(2):153169. doi:10.1080/07399330903052145.

51. Stewart RC, Umar E, Tomenson B, Creed F. Validation of the multi-dimensional scale of perceived social support (MSPSS) and the relationship between social support, intimate partner violence and antenatal depression in Malawi. BMC Psychiatry. 2014;14(1):1-23. doi:10.1186/1471-244X-14-180. 
52. Nakigudde J, Musisi S, Ehnvall A, Airaksinen E, Agren H. 54. Monticone M, Nava C, Leggero V, et al. Measurement properties Adaptation of the multidimensional scale of perceived social support in a Ugandan setting. Afr Health Sci. 2009;9 Suppl 1:S35-S41.

53. Mokkink LB, Terwee CB, Knol DL, et al. The COSMIN checklist of translated versions of the Scoliosis Research Society-22 Patient Questionnaire, SRS-22: a systematic review. Qual Life Res. 2015;24:1981-1998. doi:10.1007/s11136-015-0935-5. for evaluating the methodological quality of studies on measurement properties: a clarification of its content. BMC Med Res Methodol. 2010;10(1):22. doi:10.1186/1471-2288-10-22. 